\title{
Comparação da contenção farmacológica com cetamina e xilazina, administradas pela via intramuscular no membro torácico ou pélvico, em jacarés-do-papo-amarelo juvenis ${ }^{1}$
}

\author{
Daniela Campagnol ${ }^{2,3 *}$, Fernanda R. Lemos ${ }^{2}$, Eduardo L.F. Silva² ${ }^{2}$ João L. Rossi Júnioror ${ }^{2,3,4}$ \\ e Thatiane C. Borlini ${ }^{4}$
}

\begin{abstract}
Campagnol D., Lemos F.R., Silva E.L.F., Rossi Júnior J.L. \& Borlini T.C. 2014. [Comparison of pharmacological restraint with ketamine and xylazine, administered intramuscularly in the forelimb or hindlimb, in broad-snouted caiman juveniles.] Comparação da contenção farmacológica com cetamina e xilazina, administradas pela via intramuscular no membro torácico ou pélvico, em jacarés-do-papo-amarelo juvenis. Pesquisa Veterinária Brasileira 34(7):675-681. Programa de Mestrado em Ciência Animal, Universidade Vila Velha, Av. Comissário José Dantas de Melo 21, Boa Vista, Vila Velha, ES 29102-920, Brazil. E-mail: daniela.campagnol@uvv.br

Reptiles possess a renal portal system which can divert part of the blood from the caudal portions of the body to the kidney before it reaches the systemic circulation. In view of this, it has been recommended the administration of injectable medications in the forelimbs, in order to avoid immediate glomerular filtration, which might result in a reduction of the expected effect. The aim of this study was to compare qualitative and quantitative aspects of the pharmacological restraint provided by the combination of ketamine (30 $\mathrm{mg} /$ $\mathrm{kg}$ ) and xylazine $(1 \mathrm{mg} / \mathrm{kg})$, injected into the forelimb or hindlimb, in broad-snouted caiman juveniles (Caiman latirostris). Eight male animals, with a mean weight ( \pm SD) of 1.3 $( \pm 0.3) \mathrm{kg}$, and aged about 2 years old, were anesthetized on two separate occasions with an interval of 7 days. On each occasion, the animals were randomly assigned to receive the anesthetic combination intramuscularly into the forelimb (FL treatment) or hindlimb (HL treatment). The time intervals between administration of treatment and loss of the righting reflex (induction time), between the loss and return of this reflex (duration of important clinical effect), and between the return of the righting reflex and first movements of ambulation (duration of residual effect) were measured as well as heart and respiratory rates and cloacal and environmental temperatures. Sedation/anesthesia scores were evaluated using a scale ranging from 0 (alert/conscious) to 10 (deep anesthesia/overdose). In the HL treatment, loss of righting reflex was not observed in two animals. Considering only those animals whose loss of righting reflex was observed, the induction time $(21 \pm 9$ and $17 \pm 5$ minutes), the duration of important clinical effect ( $35 \pm 19$ and $43 \pm 21$ minutes), and the duration of residual effect ( $28 \pm 31$ and $12 \pm 11$ minutes) were similar between the FL and HL treatments, respectively (mean $\pm \mathrm{SD}$ ). Sedation/anesthesia scores were significantly higher than at baseline from 20 to 50 minutes in both treatments and, at 30 minutes (peak sedative effect), the median score (interquartile range) was 3.5(2.3-4.0) in the FL treatment and 3.0(2.0-4.0) in the HL treatment. Differences between treatments in physiological variables were not observed. In both treatments, withdrawal reflex in response to digital clamping
\end{abstract}

\footnotetext{
${ }^{1}$ Recebido em 6 de dezembro de 2013.

Aceito para publicação em 10 de junho de 2014.

${ }^{2}$ Programa de Mestrado em Ciência Animal, Universidade Vila Velha, Av. Comissário José Dantas de Melo 21, Boa Vista, Vila Velha, ES 29102-920, Brasil. E-mails: fe_rlemos@hotmail.com; joao.rossi@uvv.br*Autor para correspondência: daniela.campagnol@uvv.br
}

\footnotetext{
${ }^{3}$ Curso de Medicina Veterinária, Universidade Vila Velha, Av. Comissário José Dantas de Melo 21, Boa Vista, Vila Velha, ES. E-mails: eduardo_lfs@ yahoo.com.br

${ }^{4}$ Programa de Mestrado em Ecologia de Ecossistemas, Universidade Vila Velha, Av. Comissário José Dantas de Melo 21, Boa Vista, Vila Velha, ES. E-mail: thatianeborlini@hotmail.com
} 
was not absent at any timepoint. The administration of xylazine-ketamine combination in the forelimb or hindlimb of broad-snouted caiman juveniles provides similar effects, suggesting that the influence of the renal portal system is not clinically relevant. At the doses used, such combination does not induce surgical anesthesia and the immobility/pharmacological restraint is unpredictable and of short duration.

INDEX TERMS: Renal portal system, ketamine, xylazine, dissociative anesthetics, alpha-2 agonists, chemical restraint, sedation, broad-snouted caiman, Caiman latirostris, crocodilians.

RESUMO.- Os répteis possuem um sistema porta-renal, o qual pode desviar parte do sangue proveniente das porções caudais do corpo aos rins antes que a mesma atinja a circulação sistêmica. Em vista disto, vem sendo aconselhada a administração de medicamentos injetáveis nos membros torácicos, para que se evite a filtração imediata pelo parênquima renal, causando redução do efeito esperado. 0 objetivo do presente estudo foi comparar aspectos qualitativos e quantitativos da associação de cetamina (30 mg/kg) e xilazina (1 mg/kg), injetada no membro torácico ou pélvico, em jacarés-do-papo-amarelo (Caiman latirostris) juvenis. Oito animais machos com peso médio $( \pm \mathrm{DP})$ de $1,3( \pm 0,3) \mathrm{kg}$ e, aproximadamente, dois anos de idade foram anestesiados em duas ocasiões distintas com intervalo de sete dias. Em cada ocasião, os animais receberam, de forma aleatória, a associação anestésica por via intramuscular em membro torácico (tratamento MT) ou pélvico (tratamento MP). Foram avaliados os intervalos de tempo entre a administração do tratamento e a perda do reflexo de endireitamento (período de indução), entre a perda e o retorno desse reflexo (duração do efeito clínico importante) e entre o retorno do reflexo de endireitamento e os primeiros movimentos de deambulação (duração do efeito residual), as frequências cardíaca e respiratória e as temperaturas ambiental e cloacal. Os escores de sedação/anestesia foram avaliados através de uma escala com variação de 0 (alerta/consciente) a 10 (anestesia profunda/sobredosagem). No tratamento MP, dois animais não apresentaram perda de reflexo de endireitamento. Considerando somente aqueles que apresentaram

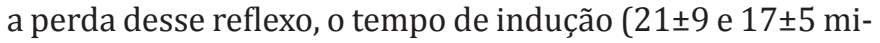
nutos) e a duração do efeito clínico importante $(35 \pm 19$ e $43 \pm 21$ minutos) e residual (28 \pm 31 e $12 \pm 11$ minutos) foram similares entre os tratamentos MT e MP (média \pm desvio padrão). 0 escore de sedação foi significativamente maior que o basal de 20 a 50 minutos nos dois tratamentos e, aos 30 minutos (pico de efeito), o escore mediano (interquartil) foi 3,5(2,3-4,0) no tratamento MT e 3,0(2,0-4,0) no tratamento MP. Assim como nos escores de sedação, diferenças entre tratamentos nas variáveis fisiológicas não foram observadas. Em ambos os tratamentos, o reflexo de retirada de membro ao pinçamento digital não foi abolido. A administração da associação de xilazina e cetamina em membro pélvico ou torácico de jacarés-do-papo-amarelo juvenis promove efeitos similares, sugerindo que a influência do sistema porta-renal não é clinicamente relevante. Nas doses empregadas, tal protocolo não promove anestesia cirúrgica e a imobilidade/contenção farmacológica é imprevisível e de curta duração.

TERMOS DE INDEXAÇÃO: Sistema portal-renal, cetamina, xilazina, anestésicos dissociativos, agonistas alfa-2, contenção química, sedação, jacarés-do-papo-amarelo, Caiman latirostris, crocodilianos.

\section{INTRODUÇÃO}

Proporcionar anestesia adequada em répteis ainda é considerado um grande desafio. Dificuldades na avaliação da profundidade anestésica e monitoração dos paramentos vitais e a escassez de informações quanto à fisiologia da dor e às terapias analgésicas nessa classe, são fatores que influenciam negativamente na qualidade da anestesia (Read, 2004). Em um estudo prospectivo, a taxa de mortalidade perianestésica em répteis, apesar do número restrito de animais avaliados (1,49\%; 2 óbitos/134 animais), foi substancialmente maior que a observada em cães $(0,17 \%)$ e gatos $(0,24 \%)$ (Brodbelt et al. 2008).

Os répteis apresentam metabolismo lento e necessitam de fontes de calor externas para regular a sua temperatura corpórea e sua taxa metabólica. Dessa forma, tanto a absorção quanto a depuração de fármacos empregados na contenção farmacológica/anestesia desses animais pode ser intensamente retardada em condições térmicas abaixo da zona de temperatura ideal (Fleming 2007, Heard \& Stetter 2007). Outra particularidade dos répteis é a presença do sistema porta-renal. Esse sistema permite que o sangue proveniente da parte caudal do corpo seja direcionado para os rins antes que atinja a circulação central (Skypes \& Greenacre 2006). Foi relatado em tartarugas-do-ouvido-vermelho (Trachemys scripta elegans) que o sangue da parte caudal do corpo pode ser desviado dos rins pela abertura de uma válvula porta-renal, direcionando o sangue ao fígado, enquanto que o fechamento da válvula favorece a perfusão renal (Holz et al. 1997a). Como resultado, fármacos administrados na parte caudal do corpo podem sofrer excreção renal ou depuração hepática (metabolismo de primeira passagem) antes de atingir a circulação sistêmica, o que poderia causar diminuição no efeito esperado de agentes anestésicos (Skypes \& Greenacre 2006).

Embora seja aconselhada a administração de medicamentos injetáveis em partes corpóreas mais craniais (como os membros torácicos), em algumas espécies de répteis, essa preocupação tem se mostrado mais teórica do que prática, já que apenas uma pequena parte do sangue proveniente da cauda e membros pélvicos passa por esse sistema (Benson \& Forrest 1999). Em iguanas verdes (Iguana iguana), por exemplo, foi constatado que o sangue proveniente dos membros pélvicos é desviado para a veia cava caudal, não passando pelo sistema porta-renal (Benson \& Forrest 1999). Em tartarugas-de-orelha-vermelha (Trachemys scripta elegans), as diferenças farmacocinéticas da gentamicina e carbenicilina administradas em membro pélvico, quando comparada à promovida pela injeção em membro torácico, foram clinicamente insignificantes (Holz et al. 1997b). Em estudo mais recente realizado em crocodilos- 
-do-estuário (Crocodylus porosus), a administração de medetomidina em membro pélvico e cauda não resultou em imobilização, ao passo que a administração da mesma dose em membro torácico aboliu o reflexo de endireitamento em todos os animais avaliados (Olsson \& Phalen 2012).

Em vista do temperamento agressivo e da dificuldade anatômica de estabelecimento de acesso venoso, muitos protocolos de contenção farmacológica/anestesia em crocodilianos são administrados pela via intramuscular (IM). Este fato, somado à ampla margem de segurança, justificam a utilização frequente de agentes dissociativos (cetamina e tiletamina) nesta ordem de animais. Diferentes associações de anestésicos dissociativos, agonistas alfa- 2 adrenérgicos, benzodiazepínicos, opioides e bloqueadores neuromusculares foram propostos para uso em crocodiliano, mas há pouca informação direcionada para cada espécie (Bennett 1991, Fleming 2007, Heard \& Stetter 2007). As doses de cetamina sugeridas pela literatura para a promoção de anestesia/imobilização de crocodilianos variam amplamente $(11-110 \mathrm{mg} / \mathrm{kg})$, assim como variam a eficácia e a intensidade do seu efeito (Bennett 1991, Fleming 2007). Em estudo recente realizado em jacaretinga (Caiman crocodilus), dose isolada de $20 \mathrm{mg} / \mathrm{kg}$ de cetamina por via IM não promoveu anestesia, mas tornou os animais mais letárgicos e diminuiu a agressividade e o comportamento de fuga (Hirano 2011). Doses mais elevadas (45-70 mg/kg, IM), embora tenham promovido depressão do nível de consciência e imobilidade em jacarés-americanos (Alligator mississippiensis), não foram associadas a relaxamento muscular satisfatório e resultaram em recuperação prolongada (Terpin et al. 1978). Nessa mesma espécie e em crocodilos-do-Nilo (Crocodylus nilocutis), a associação de xilazina $(1-2 \mathrm{mg} / \mathrm{kg}$, IM) ou medetomidina $(131-220 \mu \mathrm{g} / \mathrm{kg}$ IM) a doses relativamente baixas de cetamina $(7,5-20 \mathrm{mg} / \mathrm{kg}$, IM), resultou em anestesia cirúrgica sugerindo que a inclusão do agonistas alfa-2 no protocolo promove efeito sinérgico de importância clínica (Idowu \& Akinrinmade 1986, Heaton-Jones et al. 2002).

Diante da carência de estudos em jacarés-do-papo-amarelo (Caiman latirostris), espécie geograficamente encontrada em território brasileiro, o presente estudo teve como objetivo comparar aspectos qualitativos (intensidade do efeito) e quantitativos (período de indução, duração de ação e período de recuperação) da contenção farmacológica promovida pela associação de xilazina e cetamina aplicada em membro pélvico ou torácico. A hipótese do presente estudo foi que o local de aplicação da associação influenciaria a qualidade e duração do efeito anestésico.

\section{MATERIAL E MÉTODOS}

O estudo foi aprovado pela Comissão de Ética, Bioética e Bem Estar Animal (CEUA) da instituição (parecer no 204/2011) e pelo Sistema de Autorização e Informação em Biodiversidade (SISBIO) do Ministério do Meio Ambiente (Registro no 31270-1). Oito jacarés-do-papo-amarelo (Caiman latirostris) machos, com idade aproximada de 2 anos [comprimento médio $( \pm \mathrm{DP})$ de $73( \pm 4)$ $\mathrm{cm}]$ e peso médio $( \pm \mathrm{DP})$ de $1,3( \pm 0,3) \mathrm{kg}$, oriundos do criadouro “Caiman" em Itarana/Brasil (Licença no 3202.6938/2012), foram selecionados para o estudo. Durante a seleção, os animais foram examinados e identificados com brincos numerados fixados na escama dorsal da cauda. Animais com alterações anátomo-fisiológicas detectadas no exame físico foram excluídos do estudo. Os animais não foram privados de alimento ou água antes dos procedimentos anestésicos e todos foram, sequencialmente, retirados do seu recinto habitual com auxílio de um puçá, pesados em balança digital, medidos com fita métrica e alocados no local onde se realizaria o estudo, no mínimo, 30 minutos antes do registro dos parâmetros basais. Durante o período experimental, os animais permaneceram soltos em uma área delimitada por grades, com a mandíbula imobilizada junto à maxila com mordaça de fita adesiva, e o contato visual entre eles e os membros da equipe não foi impedido.

Cada animal foi anestesiado em duas ocasiões distintas, com intervalo de sete dias. As anestesias foram realizadas no mês de julho, entre o período de 10:00 e 17:00 horas, sendo que cada animal foi anestesiado em um período similar nos dois dias experimentais. Como protocolo anestésico, foi utilizada a associação de $30 \mathrm{mg} / \mathrm{kg}$ de cetamina a 10\% (Dopalen, Vetbrands) e 1mg/kg de xilazina a 1\%. Em cada dia experimental, $1 \mathrm{~mL}$ da formulação comercial de xilazina a 10\% (Sedazine, Fort Dodge) foi diluído em $9 \mathrm{~mL}$ de solução $\mathrm{NaCl}$ a $0,9 \%$ e homogeneizado manualmente (concentração final igual a 1\%). A associação anestésica foi administrada de forma aleatória na musculatura caudal do antebraço (membro torácico; tratamento MT) ou da coxa (membro pélvico; tratamento MP).

Após a administração do tratamento, foram registrados os intervalos entre a aplicação dos fármacos e a perda do reflexo de endireitamento (período de indução), entre a perda e o retorno do reflexo de endireitamento (duração de ação de um efeito clínico importante) e entre o retorno do reflexo de endireitamento e os primeiros movimentos voluntários de deambulação (duração de ação do efeito residual). 0 período de recuperação foi considerado o intervalo entre a aplicação dos fármacos e os primeiros movimentos de deambulação (somatória dos três intervalos anteriormente citados). Para a avaliação do reflexo de endireitamento, os animais foram posicionados em decúbito dorsal e imediatamente liberados. 0 reflexo de endireitamento foi considerado normal quando o animal assumia a posição quadrupedal prontamente (1-2 segundos); diminuído quando o animal recuperava a posição quadrupedal em um período inferior a 1 minuto, mas não prontamente; e ausente quando o animal se mantinha imóvel no decúbito dorsal durante um período superior a 1 minuto.

Para a avaliação do grau de sedação/anestesia foi proposta uma escala (Quadro 1) baseada em sinais clínicos utilizados para monitoração do plano anestésico em répteis (Malley 1997). De acordo com essa escala, a soma da pontuação igual a "0" (zero) representa o animal alerta/consciente"; 1 e 2", sedação discreta; "3 e 4", sedação moderada; "5 e 6", sedação profunda/anestesia superficial; "7 e 8", anestesia cirúrgica; "9 a 10" anestesia profunda/sobredosagem.

Movimentos voluntários foram verificados durante os dois minutos antecedentes a cada momento de avaliação, sem que houvesse contato físico entre o avaliador e o animal. Para avaliar a capacidade de sustentação da cabeça, a mesma foi elevada e imediatamente liberada, sendo considerada positiva se o animal não retornasse a apoiar a cabeça na superfície imediatamente. 0 reflexo palpebral foi avaliado através do toque leve na comissura medial da pálpebra com uma haste de algodão umedecida em solução de $\mathrm{NaCl}$ a $0,9 \%$. O reflexo corneal, caracterizado pela movimentação da terceira pálpebra em resposta ao toque da haste na córnea, somente foi verificado na ausência do reflexo palpebral. Caso contrário, o reflexo corneal foi automaticamente considerado presente. A presença do reflexo digital foi avaliada com pinça hemostática Kelly, cuja extremidade foi protegida com 
Quadro 1. Escala para avaliação do grau de sedação/anestesia em jacarés-do-papo-amarelo

\begin{tabular}{lc}
\hline Parâmetros avaliados & Escore \\
\hline Movimentos voluntários & 0 - Presente \\
Capacidade de sustentação de cabeça & 1 - Ausente \\
& 0 - Presente \\
Reflexo de endireitamento & 1 - Ausente \\
& 0 - Normal \\
Reflexo ao pinçamento digital no membro torácico & 1 - Deprimido \\
& 2 - Ausente \\
Reflexo ao pinçamento digital no membro pélvico & 0 - Normal \\
& 1 - Deprimido \\
Reflexo palpebral & 2 - Ausente \\
& 0 - Normal \\
Reflexo corneal & 1 - Deprimido \\
& 2 -Ausente \\
Total de pontos & 0 - Presente \\
& 1 - Ausente \\
& 0 - Presente \\
& 1 - Ausente \\
& 0 - 10
\end{tabular}

fita adesiva a fim de impedir/minimizar lesões teciduais. 0 fechamento da pinça foi realizado gradualmente e interrompido imediatamente antes que a primeira cremalheira fosse atingida (compressão máxima) ou que movimento de retirada do membro fosse observado. 0 reflexo digital foi considerado normal quando o animal prontamente apresentou resposta motora frente a uma leve compressão da pinça; deprimido quando o animal apresentou resposta motora ao estímulo máximo em um tempo inferior ou igual a 3 segundos; e ausente quando o animal não apresentou resposta motora ao estímulo máximo em um tempo superior a 3 segundos.

Durante todo o período de avaliação, os animais foram acomodados sobre um colchão termoelétrico (Ortovet), cuja temperatura foi regulada de forma similar em todas as anestesias. Um doppler vascular (DVT 600, Martec) foi empregado para monitoração da frequência cardíaca (FC), posicionando o sensor de fluxo na porção médio-ventral do corpo do animal. A frequência respiratória (FR) foi obtida por observação direta dos movimentos tóraco-abdominais. As temperaturas cloacal e ambiental foram mensuradas através de um termômetro digital (termômetro máxima e mínima, Incoterm).

O escore de sedação foi avaliado antes (momento basal) e após $10,20,30,40,50$ e 60 minutos da administração do tratamento experimental, enquanto que a FC, a FR e as temperaturas foram registradas no momento basal e após $20,30,40,50$ e 60 minutos do tratamento. A exceção do reflexo ao pinçamento de dígito, os demais parâmetros da escala de sedação foram avaliados antes do registro das variáveis fisiológicas. Todas as avaliações foram realizadas por um observador que desconhecia o local de aplicação do protocolo anestésico.

Para verificar se os dados apresentaram distribuição normal foi utilizado o teste de Kolmogorov-Smirnov. Para a comparação dos escores de sedação mensurados ao longo do tempo em relação ao escore basal foi utilizado o teste de Friedman para medida repetida seguido pelo teste de Dunn. A comparação dos escores de sedação entre tratamentos foi feita através do teste de Wilcoxon. Para comparação das variáveis fisiológicas (FC, FR e temperaturas) foi utilizada a análise de variância (ANOVA) seguida pela correção de Bonferroni entre tratamentos, e pelo teste de Dunnett ao longo do tempo dentro de cada tratamento (em relação ao basal). Os parâmetros relacionados a tempo (período de indução, duração do efeito e período de recuperação) foram comparados entre tratamentos através do teste $\mathrm{t}$ pareado. 0 teste exato de Fisher foi usado para comparar o número de animais em cada tratamento que apresentaram ou não a perda do reflexo de endireitamento. As diferenças foram consideradas significativas quando $P$ foi inferior a 0,05 .

\section{RESULTADOS}

Em 2/8 animais do tratamento MP e 0/8 animais no tratamento MT não foi observada a perda do reflexo de endireitamento, mas essa diferença não foi significativa. Devido à manutenção do reflexo de endireitamento em $2 / 8$ animais no tratamento MP, à exceção do tempo de recuperação, os demais dados relacionados a tempo nesse tratamento referem-se a 6 animais. Tanto o período de indução ( $2 \pm 9$ e $17 \pm 5$ minutos) quanto a duração do efeito clínico importante ( $35 \pm 19$ e $43 \pm 21$ minutos) e residual $(28 \pm 31$ e $12 \pm 11$ minutos) da associação de xilazina e cetamina foram similares independentemente do local de aplicação. Também não houve diferença significativa entre tratamentos no período de recuperação dos primeiros movimentos de deambulação, o qual foi de $84 \pm 37$ minutos no tratamento MT e de $72 \pm 19$ minutos no tratamento MP.

No momento basal, todos os animais receberam escore de sedação/anestesia igual a 0 ("zero"). A administração do protocolo em membro pélvico ou torácico resultou em escores de sedação similares entre si e significativamente maiores que o escore basal de 20 a 50 minutos (Quadro 2). De acordo com a escala empregada, o maior escore observado foi "4" em ambos os tratamentos (5/8 e 4/8 animais no tratamento MT e MP) e o pico de efeito foi observado após 30 minutos da administração dos fármacos. Em nenhum dos animais o protocolo aboliu o reflexo digital e palpebral.

Em ambos os tratamentos houve elevação significativa da FR em relação ao valor basal de 20 a 60 minutos, enquanto os valores de FC permaneceram estáveis (Quadro 3). Diferenças entre tratamentos nos valores de FR e FC não foram observadas. A temperatura ambiental foi similar entre os tratamentos e, em ambos, permaneceu estável ao longo do período experimental. No tratamento MT, houve elevação significativa da temperatura cloacal em relação ao valor basal dos 20 aos 60 minutos. A temperatura cloacal no tratamento MP permaneceu estável ao longo do tempo e diferenças significativas entre tratamentos também não foram observadas nessa variável (Quadro 4).

Quadro 2. Valores medianos (intervalos interquartis) dos escores de sedação/anestesia ( 0 a 10 pontos) registrados antes (basal) e após a administração intramuscular de xilazina (1mg/ $\mathrm{kg}$ ) e cetamina $(30 \mathrm{mg} / \mathrm{kg}$ ) no membro torácico (tratamento MT) ou pélvico (tratamento MP) em 8 jacarés do papo amarelo

\begin{tabular}{lcc}
\hline & \multicolumn{2}{c}{ Tratamentos } \\
\cline { 2 - 3 } & \multicolumn{1}{c}{ MT } & MP \\
\hline Basal & $0,0(0,0-0,0)$ & $0,0(0,0-0,0)$ \\
$10 \mathrm{~min}$ & $1,5(1,0-2,8)$ & $1,0(0,0-1,8)$ \\
$20 \mathrm{~min}$ & $3,0(1,3-4,0)^{*}$ & $3,0(1,3-3,8)^{*}$ \\
$30 \mathrm{~min}$ & $3,5(2,3-4,0)^{*}$ & $3,0(2,0-4,0)^{*}$ \\
$40 \mathrm{~min}$ & $3,5(2,3-4,0)^{*}$ & $2,5(1,3-3,8)^{*}$ \\
$50 \mathrm{~min}$ & $3,0(0,5-3,0)^{*}$ & $2,5(1,3-3,8)^{*}$ \\
$60 \mathrm{~min}$ & $2,0(0,3-2,0)$ & $2,0(0,0-3,0)$
\end{tabular}

* Diferença significativa em relação ao basal (teste de Dunn; $\mathrm{P}<0,05$ ). 
Quadro 3. Valores médios \pm desvios padrão da frequência cardíaca (FC) e frequência respiratória (FR) registrados antes (basal) e após a administração intramuscular de xilazina $(1 \mathrm{mg} / \mathrm{kg})$ e cetamina $(30 \mathrm{mg} / \mathrm{kg})$ no membro torácico (tratamento MT) ou pélvico (tratamento MP) em 8 jacarés do papo amarelo

\begin{tabular}{cccc}
\hline & \multicolumn{3}{c}{ Tratamentos } \\
\cline { 2 - 4 } FC (bpm) & MT & MP \\
& Basal & $54 \pm 5$ & $54 \pm 9$ \\
& $20 \mathrm{~min}$ & $53 \pm 7$ & $57 \pm 8$ \\
& $30 \mathrm{~min}$ & $54 \pm 5$ & $57 \pm 8$ \\
& $40 \mathrm{~min}$ & $56 \pm 5$ & $57 \pm 7$ \\
FR (mpm) & $50 \mathrm{~min}$ & $58 \pm 5$ & $56 \pm 6$ \\
& $60 \mathrm{~min}$ & $58 \pm 4$ & $57 \pm 6$ \\
& Basal & $8 \pm 5$ & $8 \pm 5$ \\
& $20 \mathrm{~min}$ & $21 \pm 3^{*}$ & $20 \pm 8^{*}$ \\
& $30 \mathrm{~min}$ & $19 \pm 9^{*}$ & $20 \pm 7^{*}$ \\
& $40 \mathrm{~min}$ & $20 \pm 4^{*}$ & $18 \pm 6^{*}$ \\
& $50 \mathrm{~min}$ & $17 \pm 8^{*}$ & $17 \pm 9^{*}$ \\
$60 \mathrm{~min}$ & $17 \pm 8^{*}$ & $16 \pm 7^{*}$
\end{tabular}

* Diferença significativa em relação ao valor basal (teste de Dunnett; $\mathrm{P}<0,05$ ).

Quadro 4. Valores médios \pm desvios padrão da temperatura cloacal/ambiental $\left({ }^{\circ} \mathrm{C}\right)$ registrados antes (basal) e após a administração intramuscular de xilazina $(1 \mathrm{mg} / \mathrm{kg})$ e cetamina $(30 \mathrm{mg} / \mathrm{kg})$ no membro torácico (tratamento MT) ou pélvico (tratamento MP) em 8 jacarés do papo amarelo

\begin{tabular}{lcc}
\hline & \multicolumn{2}{c}{ Tratamentos } \\
\cline { 2 - 3 } & MT & MP \\
\hline Basal & $27,7 \pm 0,7 / 28,0 \pm 1,7$ & $28,6 \pm 2,4 / 27,1 \pm 0,6$ \\
$20 \mathrm{~min}$ & $28,8 \pm 0,7^{*} / 27,5 \pm 1,3$ & $29,1 \pm 1,7 / 27,5 \pm 1,6$ \\
$30 \mathrm{~min}$ & $28,9 \pm 0,8^{*} / 27,8 \pm 1,5$ & $28,7 \pm 1,9 / 27,5 \pm 1,7$ \\
$40 \mathrm{~min}$ & $29,2 \pm 0,6^{*} / 27,3 \pm 1,4$ & $29,1 \pm 1,8 / 27,5 \pm 1,5$ \\
$50 \mathrm{~min}$ & $29,0 \pm 0,7^{*} / 27,6 \pm 1,4$ & $28,8 \pm 1,9 / 27,4 \pm 1,7$ \\
$60 \mathrm{~min}$ & $29,4 \pm 0,9^{*} / 27,7 \pm 1,7$ & $28,8 \pm 1,6 / 27,5 \pm 1,9$
\end{tabular}

* Diferença significativa em relação ao basal (teste de Dunnett; $\mathrm{P}<0,05$ ).

\section{DISCUSSÃO}

No presente estudo, a intensidade do efeito promovido pelo protocolo investigado foi avaliada através de uma escala de pontos desenvolvida a partir da classificação proposta por Malley (1997). Enquanto Malley propõe uma classificação descritiva da anestesia em quatro diferentes estágios (estágio 1: ausência de relaxamento muscular, presença de movimentos voluntários lentos e reflexo de endireitamento, e resposta positiva a estímulos nociceptivos; estágio 2: relaxamento muscular moderado, presença de poucos movimentos voluntários, reflexo de endireitamento alterado e resposta positiva a estímulos nociceptivos; estágio $3 \mathrm{ou}$ estágio cirúrgico: ausência de reflexo de endireitamento e movimentos voluntários, e resposta negativa a estímulos nociceptivos; e estágio 4: anestesia profunda/sobredosagem/morte iminente), a escala proposta no presente estudo designa pontos $(0,1$ ou 2) aos mesmos sinais clínicos utilizados por Malley e classifica a intensidade do efeito através de um valor numérico resultante da somatória desses pontos. Esta mudança no sistema de classificação foi proposta com o intuito de permitir a diferenciação da intensidade do efeito dentro de um mesmo estágio anestésico e facilitar a comparação entre tratamentos. Além disso, em estudo piloto foi observada variabilidade no conjunto de características apresentadas pelos animais, onde alguns apresentavam movimentos espontâneos e/ou capacidade de sustentação da cabeça mesmo na ausência reflexo de endireitamento, gerando dúvidas quanto à classificação dos estágios anestésicos de acordo com a proposta descritiva de Malley (1997). A escala utilizada para avaliação da sedação/anestesia no presente estudo, entretanto, desconsidera as alterações autonômicas frente a estímulos dolorosos. Sendo assim, estudos adicionais são necessários para avaliar a relação dessas alterações com os estágios de anestesia e incorporá-las aos métodos de avaliação existentes.

Com a metodologia utilizada no presente estudo, a associação de $30 \mathrm{mg} / \mathrm{kg}$ de cetamina à $1 \mathrm{mg} / \mathrm{kg}$ de xilazina, administrada pela via IM, foi insuficiente para promover "anestesia cirúrgica" (escore = 7-8 pontos), uma vez que todos os animais mantiveram resposta motora ao pinçamento de dígito. Embora esse protocolo também não tenha garantido imobilidade em $100 \%$ dos animais (escore $>4$ ), a intensidade de sedação obtida se mostrou adequada para o manejo e realização de procedimentos rápidos ( $<30$ minutos) e não dolorosos na maioria dos animais. Há exceção de 2 animais, que quando submetidos ao tratamento MP apresentaram sedação discreta (escore $=1$ ) ou imperceptível (escore $=0$ ) durante os 60 minutos de avaliação, os demais se mostraram mais letárgicos e com o comportamento de luta e fuga menos evidente que o observado no momento basal. 0 fato de a mandíbula ter sido mantida imobilizada com mordaça durante todo o período experimental, entretanto, não permite afirmar que a intensidade do efeito sedativo promovido por esse protocolo seja suficiente para a intubação orotraqueal ou realização de procedimentos em cavidade oral, nem que garanta a segurança dos profissionais em contato direto com o animal sem contenção mandibular. Não foram encontrados estudos avaliando os efeitos da cetamina, isoladamente ou em associação a outros fármacos, em jacarés-do-papo-amarelo. Em jacaretingas (Caiman crocodilus), uma dose mais baixa de cetamina racêmica $(20 \mathrm{mg} / \mathrm{kg})$, isolada ou associada à $2 \mathrm{mg} / \mathrm{kg}$ de midazolam, também se mostrou suficiente somente para contenção, não sendo indicada para a intubação ou para a realização de procedimentos cirúrgicos (Hirano 2011). Em jacarés-americanos (Alligator mississipiensis), a administração de 45 a $70 \mathrm{mg} / \mathrm{kg}$ de cetamina promoveu imobilidade por 10 a 20 minutos, mas a anestesia cirúrgica somente foi obtida com a administração de doses consideravelmente maiores $(80-100 \mathrm{mg} / \mathrm{kg})$ (Bennet 1991).

A associação de um agonistas alfa- 2 adrenérgicos à cetamina, assim como nas espécies domésticas, também parece potencializar o efeito sedativo/anestésico do agente dissociativo em répteis, permitindo a utilização de dose mais reduzidas para a obtenção de efeito clínico importante (Idowu \& Akinrinmade 1986, Greer et al. 2001, Heaton-Jones et al. 2002, Pratap et al. 2006). Em serpentes (Naja naja), a associação de cetamina $(40 \mathrm{mg} / \mathrm{kg})$ e xilazina $(0,4 \mathrm{mg} / \mathrm{kg})$ foi capaz de promover anestesia cirúrgica com início após 45-50 minutos de sua administração e duração de até 240 minutos (Pratap et al. 2006). Em tartarugas-do-ouvido-vermelho (Trachemys scripta elegans), a associação de $10 \mathrm{mg} /$ 
$\mathrm{kg}$ de cetamina à $200 \mu \mathrm{g} / \mathrm{kg}$ de medetomidina, um agonista mais seletivo para receptores $\alpha_{2}$ que a xilazina, promoveu plano anestésico suficiente para a realização de incisão e sutura de pele (Greer et al. 2001). Anestesia cirúrgica com duração de 50 minutos também foi obtida em crocodilos-do-Nilo (Crocodylus niloticus) após a administração IM de $20 \mathrm{mg} / \mathrm{kg}$ de cetamina, precedida 35 minutos por $1-2 \mathrm{mg} / \mathrm{kg}$ de xilazina pela mesma via (Idowu \& Akinrinmade 1986). No presente estudo, a inclusão de $1 \mathrm{mg} / \mathrm{kg}$ de xilazina ao protocolo não parece ter intensificado nem prolongado o efeito anestésico da cetamina $(30 \mathrm{mg} / \mathrm{kg})$. Entretanto, não é possível afirmar a real contribuição da xilazina sobre o efeito observado neste estudo pelo fato de não haver um grupo controle tratado apenas com o agente dissociativo. Uma possível explicação para a falha do protocolo anestésico empregado neste estudo em proporcionar anestesia cirúrgica em jacarés-do-papo-amarelo pode estar relacionada ao fato de uma amostra juvenil ter sido utilizada. Em um estudo em jacarés-americanos (Alligator mississippiensis), os animais jovens (peso médio 6,8 kg) necessitaram de doses (média \pm desvio padrão) significativamente maiores de medetomidina $(220 \pm 77 \mu \mathrm{g} / \mathrm{kg})$ em associação à cetamina $(10,0 \pm 4,9 \mathrm{mg} / \mathrm{kg}$ ) do que animais adultos (peso médio $33,7 \mathrm{~kg}$ ), cujas doses de medetomidina e cetamina foram, respectivamente, $131 \pm 20 \mu \mathrm{g} / \mathrm{kg}$ e $7,5 \pm 4,2 \mathrm{mg} / \mathrm{kg}$ (Heaton-Jones et al. 2002).

Assim como outras espécies de répteis, os crocodilianos também apresentam um sistema porta-renal constituídos por uma veia renal, a qual recebe o sangue da metade caudal do corpo através das veias epigástrica e ilíacas externas (Fleming 2007). Apesar das particularidades anatômicas, no presente estudo, não houve diferença significativa nos escores de sedação/anestesia bem como nos períodos de indução, duração da ação e recuperação após a administração da associação xilazina-cetamina no membro torácico ou pélvico em jacarés-do-papo-amarelo. Estes dados contrariam os achados do estudo realizado por Olsson \& Phalen (2012), onde a medetomidina se mostrou incapaz de promover imobilização em crocodilos-do-estuário (Crocodylus porosus) quando administrada no membro pélvico e cauda, ao passo que sua administração em membro torácico aboliu o reflexo de endireitamento em $100 \%$ dos animais. Em um estudo realizado in vivo em galinhas (Gallus domesticus), que também apresentam sistema porta-renal, foi demonstrado que a administração de adrenalina causou aumento do fluxo sanguíneo nas veias porta hepática e cava caudal, o que poderia sugerir a abertura da válvula porta-renal (Sturkie et al. 1978).

É possível que o estresse de contenção dos animais do presente estudo tenha resultado em liberação de catecolaminas, com a consequente abertura da válvula porta-renal, reduzindo o fluxo sanguíneo renal, o que poderia explicar a ausência de diferenças nos tratamentos. Outra possível explicação, sugerida em um estudo anterior, é que, apesar da existência de inervação adrenérgica e colinérgica, a válvula porta-renal pode não apresentar importância funcional (Sturkie et al.1978) e ter sua influência sobre a excreção de fármacos superestimada. Além disso, não se pode descartar a possibilidade de um efeito direto dos próprios fárma- cos utilizados na contenção farmacológica/anestesia sobre o funcionamento do sistema porta-renal. No presente estudo, 2/8 animais do tratamento MP não apresentaram perda do reflexo de endireitamento, enquanto que, no tratamento MT, houve a perda deste reflexo em todos os animais (8/8 animais). Embora a diferença dessas proporções não tenha sido estatisticamente significativa, é possível que o poder da análise utilizada tenha sido restrito devido ao baixo número de animais empregado no estudo. Uma análise retroativa detectou que a utilização de 34 animais permitiria a detecção de diferença significativa entre os tratamentos com poder de $95 \%$, baseado nas proporções observadas nesse estudo (25\% de animais com ausência de reflexo de endireitamento no tratamento MP versus $0 \%$ no tratamento MT). Portanto, recomenda-se a realização de estudos com uma amostra maior de animais para esclarecer a influência do local de aplicação sobre o efeito anestésico da associação xilazina-cetamina.

Os animais do presente estudo apresentaram FC basal (média de $55 \mathrm{bpm}$ ) consideravelmente maior que a registrada por Hagensen et al. (2010) em exemplares da mesma espécie (média de $27 \mathrm{bpm}$ ) em temperaturas ambientais similares (aprox.. $30^{\circ} \mathrm{C}$ ). Clyde et al. (1992) relacionaram os valores elevados de FC (média de 57 bpm) ao estresse de manipulação/contenção em jacarés-americanos (American alligator), o que também pode justificar a diferença na FC obtida no presente estudo. Os valores de FR basais foram similares aos registrados em outros estudos em animais da mesma ordem (4-12 mpm) (Heaton-Jones et al. 2002), mas valores inferiores (1-4 mpm) também são relatados na literatura como sendo normais para crocodilianos. Em jacarés-americanos (Alligator mississippiensis) juvenis, a administração da associação de medetomidina $(220 \mu \mathrm{g} /$ $\mathrm{kg})$ e cetamina $(10 \mathrm{mg} / \mathrm{kg})$ causou redução significativa na FC (de 37 para $13 \mathrm{bpm}$ ) e na FR (de 8 para $5 \mathrm{mpm}$ ) (Heaton-Jones et al. 2002). Esses resultados parecem ter sido causados pelo agonistas $\alpha_{2}$ (medetomidina), uma vez que sua administração isolada causou redução acentuada da FC e FR em crocodilos-do-estuário (Crocodylus porosus) e crocodilos-de-água-doce (Crocodylus johnstoni) (Olsson \& Phalen 2012, 2013). Diferente do que foi relatado por estes autores, no presente estudo não houve alterações significativas na FC e a FR se elevou após a administração do protocolo anestésico. Em mamíferos, a medetomidina apresenta potência 5 a 10 vezes maior que a xilazina (Lemke 2007). Assumindo que a ordem de potência desses fármacos seja a mesma em répteis, a dose de xilazina equipotente a $220 \mu \mathrm{g} /$ $\mathrm{kg}$ de medetomidina deveria ser de $22 \mathrm{a} 44 \mathrm{mg} / \mathrm{kg}$ de xilazina. É possível que, a dose de xilazina utilizada no presente estudo tenha sido insuficiente para causar a depressão de tais parâmetros cardiorrespiratórios. Esta hipótese, entretanto, não explica a elevação na FR observada após a administração do tratamento. Durante a execução do estudo, notou-se maior dificuldade na visualização dos movimentos respiratórios no período pós-tratamento e uma resposta compensatória à redução da amplitude respiratória (redução do volume corrente) poderia justificar a elevação da FR. Entretanto, estudos adicionais são necessários para comprovar essa impressão clínica. 
Por se tratar de animais ectotérmicos, o metabolismo dos répteis apresenta variações de acordo com a temperatura ambiental, interferindo na absorção e depuração dos fármacos empregados (Bennet 1991). Em crocodilianos de cativeiro, é recomendado que a temperatura ambiente seja mantida entre 25 e $35^{\circ} \mathrm{C}$ (Fleming 2007). Os valores médios da temperatura ambiente no presente estudo variaram de 27,3 a $28,0^{\circ} \mathrm{C}$ no tratamento MT e de 27,1 a $27,5^{\circ} \mathrm{C}$ no tratamento MP, não havendo diferenças significativas entre os tratamentos, nem dentro de cada tratamento ao longo do tempo. Apesar de ter ocorrido elevação significativa em relação ao basal na temperatura cloacal dos animais do tratamento MT, os valores médios desta variável não diferiram significativamente entre os tratamentos em nenhum dos momentos avaliados. Esses resultados sugerem que a influência da temperatura ambiental sobre os resultados do presente estudo foi a mesma nos tratamentos MT e MP. Além da temperatura, outro fator que influencia a taxa metabólica dos répteis é a sua situação nutricional. Em jacaretingas (Caiman crocodilus), a taxa metabólica após 1-4 dias da alimentação foi significativamente maior que após 18-50 dias de jejum (Gatten Jr 1980). No presente estudo, os hábitos de manejo do criadouro não foram alterados e jejum alimentar não foi realizado. Dessa forma, os resultados do presente estudo podem não ser reproduzidos em espécimes privados de alimento. Regurgitação não foi observada em nenhum dos animais do estudo e esse fato pode estar relacionado intensidade relativamente baixa do efeito sedativo promovido.

\section{CONCLUSÕES}

Independentemente do sítio de aplicação, a associação de xilazina e cetamina, nas doses empregadas no presente estudo, não proporciona anestesia cirúrgica e nem garante a imobilidade/contenção farmacológica em jacarés-do-papo-amarelo juvenis, criados em cativeiro e não submetidos à restrição alimentar.

Nas condições em que o estudo foi realizado, a similaridade na intensidade e na duração da sedação promovida pela administração do protocolo investigado em membro torácico ou pélvico, sugere que a influência do sistema porta-renal não é clinicamente relevante.

Estudos adicionais, utilizando um número amostral maior, avaliando os efeitos de outros fármacos/protocolos e verificando a influência de fatores intrínsecos (como o estresse), são necessários para confirmar a irrelevância do sistema porta-renal no efeito clínico de agentes anestésicos.

\section{REFERÊNCIAS}

Bennet R.A. 1991. A review of anesthesia and chemical restraint in reptiles. J. Zoo Wildl. Med. 22:282-303.

Benson K.G. \& Forrest L. 1999. Characterization of the renal portal system of the common green iguana (Iguana iguana) by digital subtraction imaging. J. Zoo Wildl. Med. 30:235-241.
Brodbelt D.C., Blissitt K.J., Hammond R.A., Neath P.J., Young L.E., Pfeiffer D.U. \& Wood J.L. 2008. The risk of death: the confidential enquiry into perioperative small animal fatalities. Vet. Anaesth. Analg. 35:365-373.

Fleming G.J. 2007. Crocodilians (crocodiles, alligators, caimans, gharial), p.223-232. In: West G., Heard D \& Caulkett N. (Eds), Zoo Animal and Wildlife Immobilization and Anesthesia. Blackwell Publishing, Ames.

Gatten Jr R.E. 1980. Metabolic rates of fasting and recently fed spectacled caimans (Caiman crocodilus), Herpetologica 36:361-364.

Greer L.L., Jenne K.J. \& Diggs H.E. 2001. Medetomidine-ketamine anesthesia in red-eared slider turtles (Trachemys scripta elegans). Contemp. Top. Lab. Anim. Sci. 40:9-11.

Hagensen M.K., Abe A.S. \& Wang T. 2010. Baroreflex control of heart rate in the broad-nosed caiman Caiman latirostris is temperature dependent. Comp. Biochem. Physiol. A, Mol. Integr. Physiol. 156:458-462.

Heard D.J. \& Stetter M.D. 2007. Reptiles, amphibians, and fish, p.869-887. In: Tranquilli W.J., Thurmon J.C. \& Grimm K.A. (Eds), Lumb and Jones' Veterinary Anesthesia and Analgesia. $4^{\text {th }}$ ed. Blackwell Publishing, Ames.

Heaton-Jones T.G., Ko J.C.H. \& Heaton-Jones D.L. 2002. Evaluation of medetomidine-ketamine anesthesia with atipamezole reversal in American alligators (Alligator mississippiensis). J. Zoo Wildl. Med. 33:36-44.

Hirano L.Q.L. 2011. Contenção farmacológica de jacaré-tinga Caiman crocodiles Linnaeus, 1758 com cetamina S(+) e cetamina racêmica, isolada ou em associação com o midazolam. Dissertação de Mestrado em Ciências Veterinárias (Saúde Animal), Faculdade de Medicina Veterinária, Universidade Federal de Uberlândia, MG. 55p.

Holz P., Barker I.K., Crawshaw G.J. \& Dobson H. 1997. The anatomy and perfusion of the renal portal system in the red-eared slider (Trachemys scripta elegans). J. Zoo Wildl. Med. 28:378-385.

Holz P., Barker I.K., Burger J.P., Crawshaw G.J. \& Conlon P.D. 1997. The effect of the renal portal system on pharmacokinetic parameters in the red-eared slider (Trachemys scripta elegans). J. Zoo Wildl. Med. 28:386393.

Idowu A.L. \& Akinrinmade J.F. 1986. Xylazine and ketamine anaesthesia in captive Nile crocodile (Crocodylus niloticus). Trop. Vet. 4:139-142.

Lemke K.A. 2007. Anticholinergics and sedatives, p.203-240. In: Tranquilli W.J., Thurmon J.C. \& Grimm K.A. (Eds), Lumb and Jones' Veterinary Anesthesia and Analgesia. $4^{\text {th }}$ ed. Blackwell Publishing, Ames.

Malley D. 1997. Reptile anaesthesia and the practicing veterinarian. In Practice 19:351-368.

Olsson A. \& Phalen D. 2012. Preliminary studies of chemical immobilization of captive juvenile estuarine (Crocodylus porosus) and Australian freshwater $(C$. johnstoni) crocodiles with medetomidine and reversal with atipamezole. Vet. Anaesth. Analg. 39:345-356.

Olsson A. \& Phalen D. 2013. The effects of decreased body temperature on the onset, duration and action of medetomidine and its antagonist atipamezole in juvenile farmed estuarine crocodiles (Crocodylus porosus). Vet. Anaesth. Analg. 40:272-279.

Pratap K., Amarpal A., Kinjavdekar P., Aithal H.P. \& Pawde A.M. 2006. Xylazine-ketamine anaesthesia in snakes (Naja naja) and its reversal with atipamezole. Indian J. Anim. Sci. 76:580-581.

Read MR. 2004. Evaluation of the use of anesthesia and analgesia in reptiles. J. Am. Vet. Med. Assoc. 224:547-552.

Skypes J.M. \& Greenacre C.B. 2006. Techniques for drug delivery in reptiles and amphibians. J. Exotic Pet Med. 15:210-217.

Sturkie P.D., Dirner G. \& Gister R. 1978. Role of renal portal valve in the shunting of blood flow in renal and hepatic circulations of chickens. Comp. Biochem. Physiol. 59:95-96.

Terpin K.M., Dodson P. \& Spotila J.R. 1978. Observations on ketamine hydrochloride as an anesthetic for alligators. Copeia 1:147-148. 